\title{
Low tension studies of collagen fibres from ligaments of the human spine
}

\author{
J. S. SHAH*, M. I. V. JAYSON†, AND W. G. J. HAMPSON $\ddagger$
}

From the H. H. Wills Physics Laboratory, University of Bristol,* Department of Medicine, University of Bristol and Royal National Hospital for Rheumatic Diseases, Bath, $\uparrow$ and

Southmead and Winford Orthopaedic Hospitals, Bristol

SUMMARY On polarization microscopy collagen fibres from human cadaveric anterior longitudinal, posterior longitudinal, and interspinous ligaments show a series of transmission and extinction bands. By observing changes in this pattern on rotating the polarizing stage and on rotating the fibres a crimped structure of the fibres was deduced and its parameters were calculated. From these data the force/strain behaviour of the fibres under low tension was calculated. This corresponded closely with the results from mechanical measurement. At the same time we documented alterations in the transmission and extinction patterns while under tensile load. The results suggest that it is the crimped structure that is responsible for the high extensibility of the collagen fibres under low tension. The initial extension is by deformity of the crimp segment. This avoids risk of tearing the collagen fibres.

In life the ligaments of the human vertebral column are commonly loaded in tension. We report the force/strain behaviour of the fibres from human anterior longitudinal ligaments, posterior longitudinal ligaments, and interspinous ligaments at low tensions. The results obtained are compared with those predicted from a mechanical model. The model closely resembles the structure of the fibres deduced from separate microscopy experiments.

\section{Methods}

At autopsy the lumbar spines were removed intact from 2 previously healthy males aged 35 and 41 years, who died shortly after road traffic accidents

Accepted for publication June 30, 1976

Correspondence to Dr. M. I. V. Jayson, Department of Medicine, Bristol Royal Infirmary, Bristol BS2 8HW without spinal injury. Fibres were sampled from the anterior longitudinal, posterior longitudinal, and interspinous ligaments at the level of the 1st and 2nd lumbar vertebrae. Individual fibres were extracted by carefully teasing apart the surface layers of the ligaments with dissecting needles and then gently pulling them in the direction at right angles to the surface of the tissue. The separated fibres were stored in iso-onic saline at $-25^{\circ} \mathrm{C}$ until ready for use. Three fitres from each of the three ligaments of the two spines, making a total of 18 fibres, were subjected to the following experiments.

We placed each fibre between the crossed filters of a polarizing microscope with its long axis parallel to the axis of polarization of one of the filters. Along the length of each fibre a series of transmission and extinction bands were seen and an example is shown in Fig. 1. The stage of the polarizing microscope was

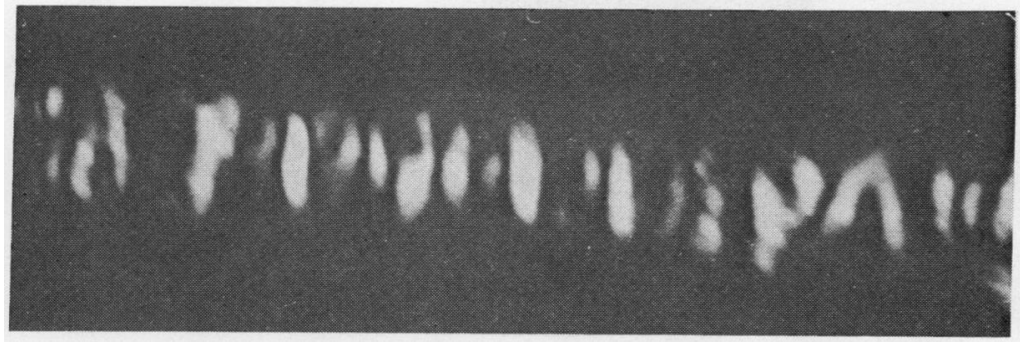

Fig. 1 Typical polarization photomicrograph showing a periodic band pattern. $\times 210$. 


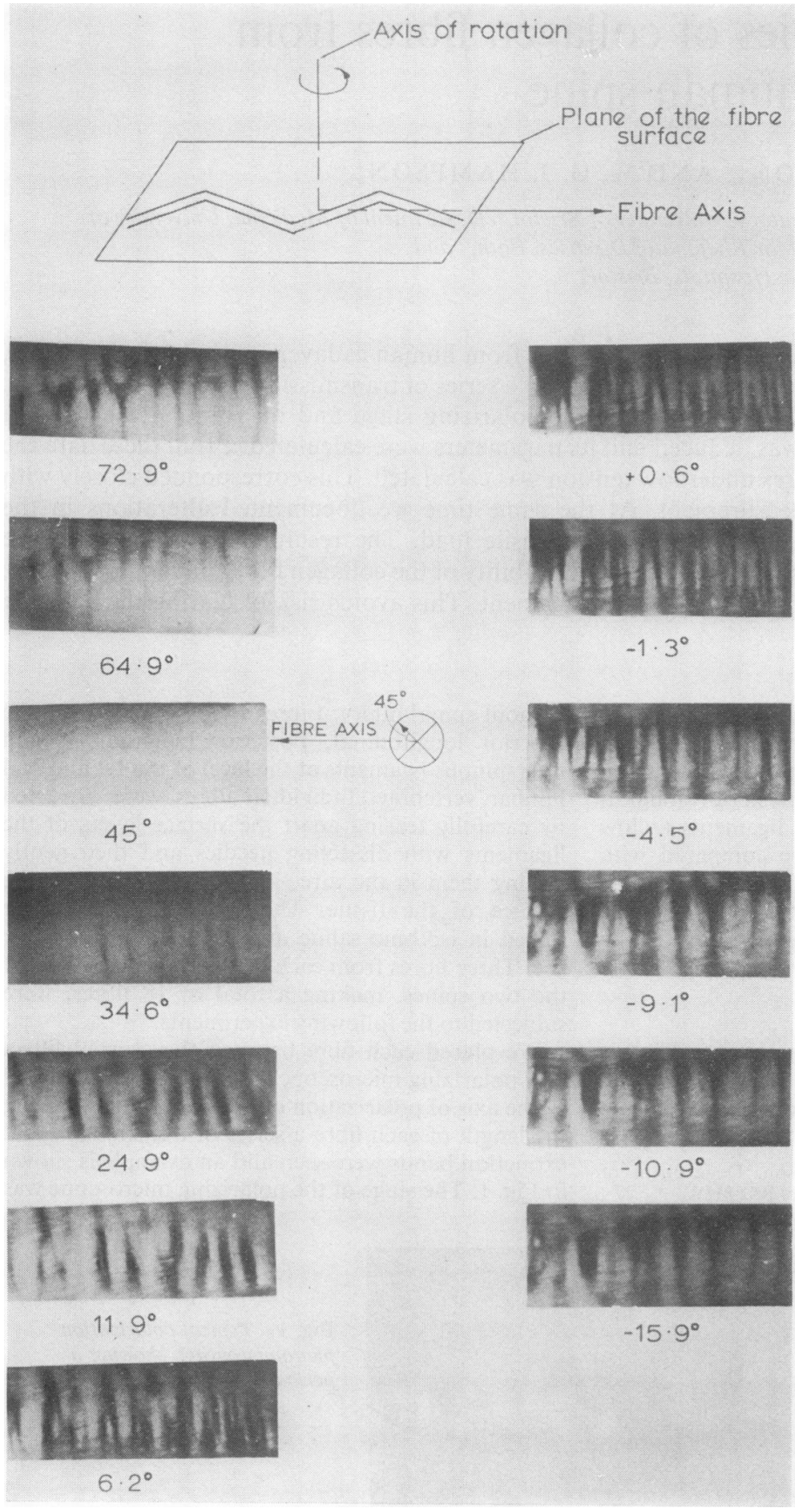

Fig. 2 Typical changes observed in the band pattern on rotation of the fibre around an axis at right. angles to the surface of the fibre. The rotation axis is diagrammatically shown at the top of the figure. The angle listed below each pattern is the rotation angle of the fibre axis, with reference to the polarizing direction of a filter, and is schematically illustrated along side one of the patterns. The crossed lines at the right angles, enclosed in the circle, are the polarizing directions of the filters: 
rotated around the axis of the microscope. At varying degrees of rotation the relative changes in the position and intensity of the bands were noted (Fig. 2). We then rotated each fibre around its own long axis and noted the changes in the band pattern. We interpreted the structure of the fibres from the band patterns by methods described by Diamant et al. (1972) and summarized in Appendix I.

In a further series of experiments we mechanically tested the fibres in a tensometer. The fibre ends were attached to the stretching heads of a Wingfield fibre tensometer (Fig. 3) by cyanoacrylic glue. Each fibre, during stretching, was immersed in isotonic saline at room temperature and was continuously viewed through a polarizing microscope. Forces up to $600 \times$ $10^{-5}$ Newtons were applied through the pulling mechanism of the tensometer at a constant strain rate of $0.4 \%$ per second. The magnitude of the applied force was measured by connecting a calibrated cantilever spring coupled direct current Linear Variable Differential Transformer (dcLVDT) type transducer to the stretching head of the tensometer. To evaluate stresses in the fibres the mean crosssectional area of each fibre was calculated from

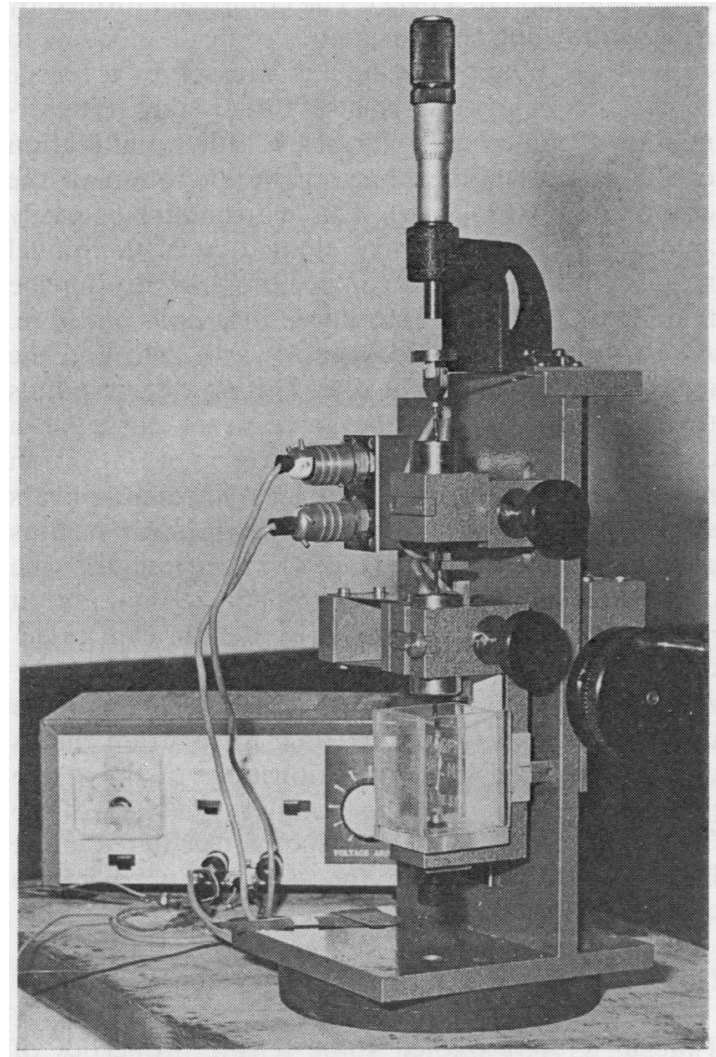

Fig. 3 Tensometer for mechanical testing of the fibres.

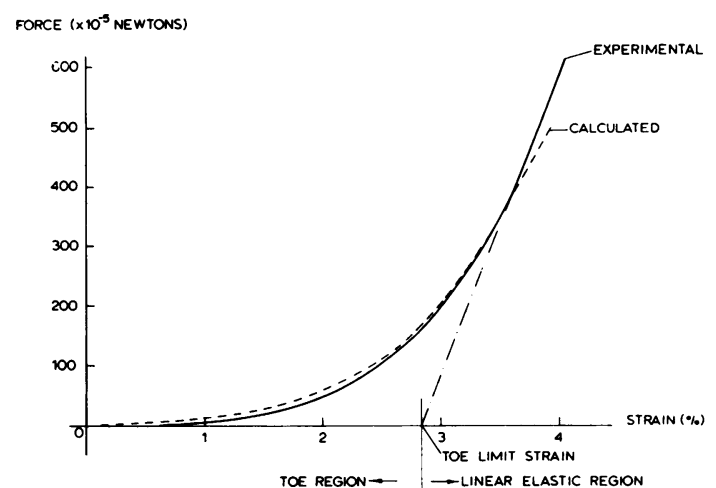

Fig. 4 A typical experiment force/strain curve (solid line). The intercept made by the extrapolation (dot-dashed line) of the linear portion with the strain axis coincides with the strain value at which the band pattern disappeared. This is defined as the toe limit strain. The curve predicted from the equation in Appendix 2 is shown as a dashed line. Conversion: SI to traditional units1 Newton $\approx 1 \mathrm{~kg} \cdot \mathrm{m} / \mathrm{s}^{2}$.

optical microscopical measurements of the fibre of two diameters at right angles to each other at ten different points along its length. Typically, the mean standard deviation of the cross-sectional area of each fibre was in the range 4-6\%. Fibre strains were measured by a dcLVDT displacement transducer. The force on the fibre and its strain were recorded in the form of a force/strain curve (Fig. 4) on an X-Y plotter. The changes in transmission and extinction bands were also noted.

\section{Results}

The changes in position of the bands (Fig. 2) on rotating the fibres were analysed as noted in Appendix I. The results suggest that all the 18 fibres examined possess a zig-zag ribbon-like or crimped structure which can be quantitatively described by the length of the crimp segment $l$ and the crimp angle $\theta$ (Fig. 5a). The values of $l$ and $\theta$ obtained by analysis of the fibres from the three ligaments are summarized in Table 1.

The values of $l$ show a large range of variation.

Table 1 Crimp parameters of fibres from human spinal ligament

\begin{tabular}{lll}
\hline & $\begin{array}{l}\text { Crimp angle } \theta^{\circ} \\
(\text { median } \pm \text { range })\end{array}$ & $\begin{array}{l}\text { Crimp length }(\mu \mathrm{m}) \\
\text { (median } \pm \text { range })\end{array}$ \\
\hline $\begin{array}{l}\text { Anterior longitudinal } \\
\text { ligament }\end{array}$ & $13.6 \pm 0.9$ & $45 \pm 4$ \\
$\begin{array}{l}\text { Posterior longitudinal } \\
\text { ligament }\end{array}$ & $15 \pm 0.4$ & $52 \pm 4$ \\
\begin{tabular}{l} 
Interspinous ligament \\
\hline
\end{tabular} & $14.8 \pm 0.5$ & $40 \pm 3$ \\
\hline
\end{tabular}




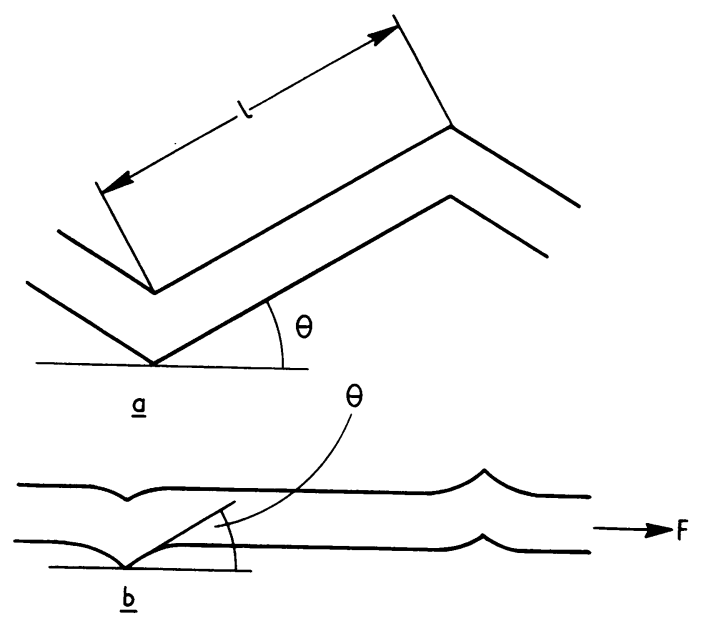

Fig. 5 (a) Schematic representation of the crimped fibre showing the crimp angle $\theta$ and the crimp segment length 1 . The figure corresponds to the undeformed model. (b) Elongation of the model. Note that, due to rigidity of attachment at the extremities, the crimp segment deforms without altering the crimp angle.

This seems to be a real variation and is not due to errors in measurement. Similar variations were also noted by Diamant et al. (1972) in rat tail tendons.

On stretching a collagen fibre there is initially a large amount of fibre elongation for the application of a relatively small force. Consequently, the initial part of the force/strain curve is nonlinear and is known as the 'toe' region. With further increment in the applied force the fibre seems to become stiffer and the linear (elastic) portion of the curve is reached. In every experiment the strain axis intercept made by the extrapolation of the linear portion of the curve (Fig. 4) corresponded to the value of the strain at which the band pattern across the fibre, seen through the polarizing microscope, disappeared. We define this value as the 'toe limit strain'. This definition of the tow limit strain emphasizes that collagen fibres exhibit additional strain over and above that expected by a linearly elastic material of the same modulus of elasticity.

Table 2 Stress-strain behaviour of fibres from human spinal ligaments (median and range)

\begin{tabular}{|c|c|c|c|}
\hline Ligament & $\begin{array}{l}\text { Toe limit } \\
\text { strain }(\%)\end{array}$ & $\begin{array}{l}\text { Stress } \\
M N / m^{2}\end{array}$ & $\begin{array}{l}\text { Modulus of } \\
\text { elasticity }\left(M N / m^{2}\right)\end{array}$ \\
\hline $\begin{array}{l}\text { Anterior longitudinal } \\
\text { ligament }\end{array}$ & $\begin{array}{l}1 \cdot 26 \\
\pm 0 \cdot 12\end{array}$ & $\begin{array}{l}0.011 \\
\pm 0.005\end{array}$ & $12 \cdot 3 \pm 1 \cdot 3$ \\
\hline $\begin{array}{l}\text { Posterior longitudinal } \\
\text { ligament }\end{array}$ & $\begin{array}{l}2 \cdot 28 \\
\pm 0.12\end{array}$ & $\begin{array}{l}0.927 \\
\pm 0.063\end{array}$ & $148 \cdot 3 \pm 22 \cdot 7$ \\
\hline Interspinous ligament & $\begin{array}{l}2.80 \\
\pm 0.49\end{array}$ & $\begin{array}{l}1.084 \\
\pm 0.090\end{array}$ & $23 \cdot 7 \pm 2 \cdot 4$ \\
\hline
\end{tabular}

See Fig. 4 for conversion factor for Newtens.
The toe limit strain values and moduli of elasticity deduced from the linear portions of the force/strain curves for the fibres studied are summarized Table 2.

INTERRELATION BETWEEN MORPHOLOGICAL AND MECHANICAL STUDIES

Diamant et al. (1972) first discovered the planar zigzag structure of collagen fibres in rat tail tendoris. The crimp structure in rat tail tendons was further confirmed by the scanning electron microscope studies of Gathercole et al. (1974), and Evans ange Barbenel (1975), and the low angle $x$-ray studies of Gathercole and Keller (1975). Here we have shovi that the fibres from the human spinal ligaments als possess a similar structure. As the polarization bandis disappear under tensile stress it is likely that the mechanical behaviour of collagen is related to ifs crimped structure.

The crimp geometry of the fibre structure loosely resembles that of a mechanical model known Elastica, first described by Euler (1744) and shown in Fig. 5a. The mechanical properties of this mode were described by Love (1944), Frisch-Fay (1962), and Diamant et al. (1972). The crimps are formed by rigidly attaching the extremities of flexible beams each other. When the model is stressed by a tensife force $F$, its elongation is substantially due to defor mation of the crimp segments, a little by alteration of the crimp length $l$, but not by alteration of the crimp angle $\theta$ (Fig. $5 b$ ). This deformation is easify achieved so that the initial elongation of the modet requires little force, i.e. it corresponds to the toe region of the force/strain curve of a collagen fibre At greater forces, $\theta$ decreases towards zero and the model is no longer applicable. This now correspond to the linear part of the force/strain curve of $\frac{a}{a}$ collagen fibre. Since the banded appearance of polarization microscopy of collagen fibres disappears at the junction between the toe and linear region of the force/strain curve (Fig. 4), it suggests that the crimp structure is maximally straightened out at that point. The strain in such a crimp chain can be des duced from the equation given in Appendix If Assuming that the human spinal ligaments are made up of multiple elastica units, their force/strain curves can be predicted from the knowledge of the crim parameters $l$ and $\theta$ and the moduli of elasticity of the fibres. A typical example of such a calculated curve is shown in Fig. 4. In the toe region it approximates closely to the experimental curve.

\section{Discussion}

Tkaczuk (1968) studied some tensile properties of the longitudinal ligaments of the human spine but 
the extents of the nonlinear regions and the values of the elastic moduli were not reported. Cronkite (1936) and Harris et al. (1966) show that the moduli of elasticity of human tendons lie in the range 28$760 \mathrm{MN} / \mathrm{m}^{2}$. The values reported in Table 2 are of the same order.

A number of studies on other collageneous tissues by Rigby et al. (1959), Abrahams (1967), Haut and Little (1972), Diamant et al. (1972), and Viidik (1973) have shown that the toe limit strains for mature tissues lie between 2 and $5 \%$, whereas in young tissues the toe region is known to extend up to $14 \%$. The values of toe limit strains for mature human spinal ligament fibres shown in Table 2 are in the range $1-3 \%$.

The qualitative correlation between the waviness of the fibres from other tissues and high extensibility in the toe region has been established by a number of workers. Lerch (1950), Verzár (1957), Cruise (1958), and Evans and Barbenel (1975) maintain that in the majority of mammalian tendons, with the possible exception of rat tail tendons, the wavy structure is due to three dimensional and approximately helical twisting of collagen fibres. Force/strain curves of the fibres, however, have not been quantitatively derived from the helical twisting structure. Dale et al. (1972) and Gathercole and Keller (1975), on the other hand, deduced that the planar waveform occurs in many mammalian and nonmammalian tissues. Our observations show that the fibres of the human spinal ligaments possess a similar planar crimp-like symmetry. Furthermore, the toe regions of the force/ strain curves of the fibres can be predicted from the observed planar structure.

Estimates of in vivo forces by Harris et al. (1964), Elliot (1965), and Gathercole and Keller (1975) suggest that the stresses normally encountered in life by collagen fibres operate in the toe region of the force/strain curve. Experimental measurements on the superficial digital flexor tendons of dogs by Shaw (1968) and on the lateral extensor tendon in sheep by Kear (1971) seem to confirm this.

Therefore our studies could indicate a way in which human intervertebral ligaments behave in life and how the crimp structure could account for the high extensibility of the spinal ligaments at low tensions. At strains within the toe region the crimp structure could act as a series of compliances capable of absorbing sudden stresses so delaying the effects of fast acting forces. In other words it could function as a tensile stress shock absorber. A sudden increase in fibre length is accommodated by alteration of the crimp structure without risk of tearing collagen fibres. Only with greater strains when the crimp structure is maximally straightened out is there risk of this occurring. This mechanism also could protect the bone, which may be important as bone is susceptible to damage at high strain rates.

This is a preliminary study showing the existence of the crimp structure and its possible significance in reference to the mechanical behaviour of the fibres in human intervertebral ligaments. Alterations in the crimp structure might be relevant to changes in the flexibility of the spine with age and when there is hypermobility such as in Marfan's and EhlersDanlos syndromes.

We thank Mr. R. G. C. Arridge for helpful discussions on the calculations, and Mr. S. Rundle for technical assistance. This work was performed with the assistance of a grant from the Arthritis and Rheumatism Council for Research.

\section{Appendix I (adapted from Diamant et al., 1972)}

Alternating extinction and transmission bands are seen on examining fibres through a polarizing microscope. This could be due to:

(1) An alternating arrangement of birefringent and nonbirefringent units along the length of the fibre.

(2) Alternate segments of the fibre behave as nonbirefringent because their optic axes* are parallel to the direction of the light (i.e. extinction due to zero birefringence).

(3) The transmitting directions of the alternating segments in the fibre are aligned to the polarization directions of each of the filters (i.e. extinction due to zero amplitude effect).

When a fibre is rotated about an axis at right angles to its long axis on polarization microscopy, the periodic band pattern remains unaltered if it is due to reasons (1) and (2). Fig. 2 shows that the positions of the bands do alter on rotation and indicates that the bands are due to periodic changes of transmission directions in the fibres (reason 3 ).

Fig. 6 schematically shows how these variations arise. The parts of the fibre transmitting light are shown as open crimp segments in contrast to the shaded segments where extinction occurs. The crossed lines enclosed in circles show the polarizing directions of the filters in relation to the fibre at different positions during rotation. In each position the angle of inclination of the fibre axis with the polarizing direction of one filter (identified by a direction marker) is shown. When the overall fibre axis is at $45^{\circ}$ to the polarizing directions of the filters the transmission directions of all the segments are equally inclined to the polarizing directions. Consequently, light is equally transmitted through the

*An optic axis is a direction in a material along which light rays do not suffer double refraction. 


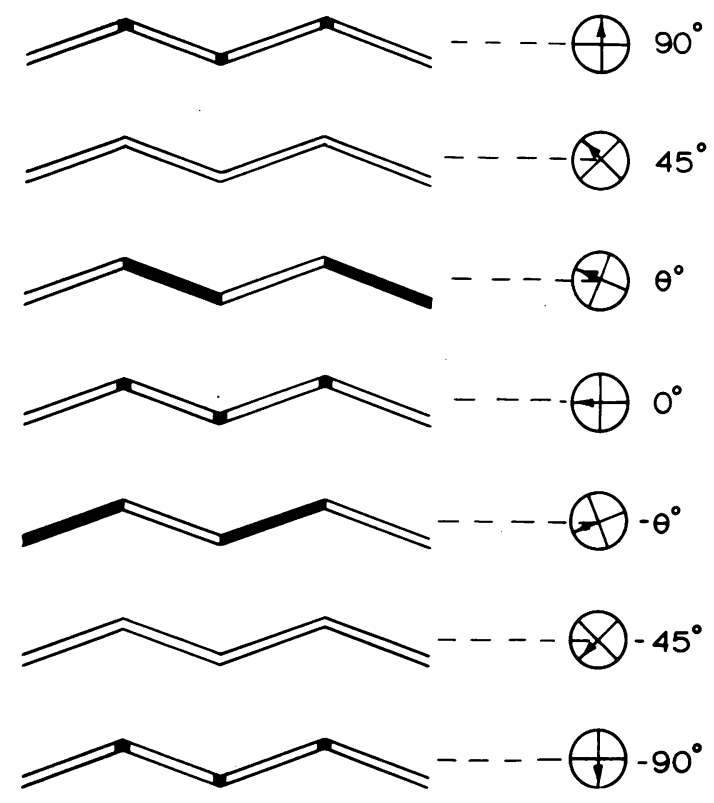

Fig. 6 Schematic changes in the extinction pattern of rotation of the fibre around an axis at right angles to the surface plane. The transmission directions of the segments along the fibre are assumed to be arranged in a zig-zag fashion. The crossed lines enclosed in circles show the polarizing directions of the filters in each position. The angle of inclination of the fibre axis with the polarizing direction of one filter (shown with a direction marker) is also shown in each position.

whole fibre and the band pattern disappears. The diminished width of the bands in certain positions indicates that the variations in transmission directions describe a sharp zig-zag rather than a gentle wavy course.

In the above tests the transmission directions on the surface are determined but it is possible that they are organized in a helical manner around the long axis of the fibre. If, however, the helical arrangement exists, then rotation of the fibre along its own axis (i.e. the axis of helicity) will cause the extinction bands to move uniformly along the fibre and in the winding direction of the helix just as we see the threads in a screw shift on rotation. Experimentally we did not see such movement in any of the fibres. Therefore the observed changes are consistent with a planar crimp arrangement.

For measuring the crimp angles a fibre is placed on the microscope stage and rotated to the position where the extinction bands are at their narrowest (i.e. the fibre axis is parallel to the direction of polarization of one of the filters). The stage is rotated first clockwise and then counterclockwise to the

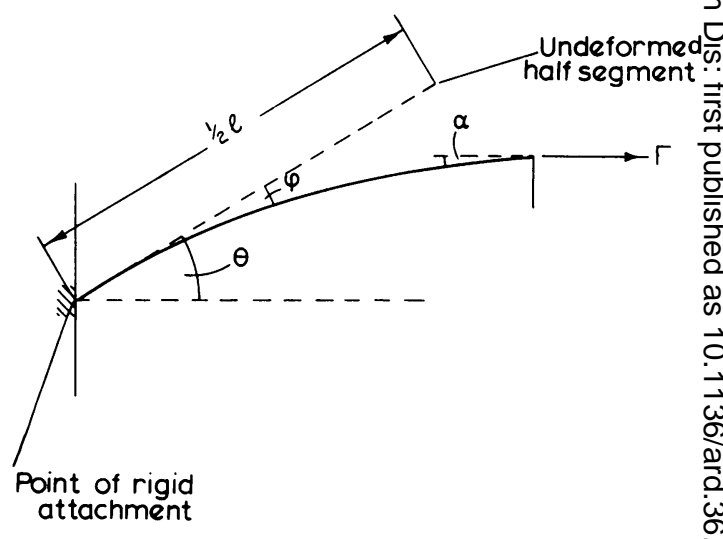

Fig. 7 Mode of deformation of half crimp segment by a force $\mathrm{F}$. The angular parameters appearing in the equation in Appendix II are shown here.

positions where they become broadest; the average of the rotation angle gives the average crimp angle?

In its broadest position the width of an extinction band is $l \cos \theta$, where $l$ is the length of the crimp segment and $\theta$ is the crimp angle.

Appendix II (from Love, 1944)

Fig. 7 shows the mode of deformation of a half crimpo segment (i.e. from apex to the centre of the segment ${ }^{\circ}$ under the influence of force $F$. The strain $\epsilon$ of the half segment under applied force $F$ is given by

$$
\epsilon=\frac{1-\cos \theta-2 \mathrm{I}+\cos \theta(1-2 \mathrm{I}) F / \mathrm{AE}}{\cos \theta-\cos \theta(1-2 \mathrm{I}) F / \mathrm{AE}}
$$

where $E=$ modulus of elasticity, $F=$ applied force $A=$ cross-sectional area of the segment, $k=$ cosi $\left(\frac{1}{2} \alpha\right), \alpha \& \varphi$ are the angles defined in Fig. 7 , and $I$ is a shortform notation for the expression

$$
\mathrm{I}=\frac{E(k)-E(k, \varphi)}{[K(k)-F(k, \varphi)]}
$$

in which $[K(k)-F(k, \varphi)]$ is a mathematical functions known as elliptical integral of the 1st kind, and $E(k, \varphi), E(k)$ are mathematical functions known aso elliptical integrals of the 2 nd kind. The values of theo above functions can be found from tables listed in standard reference books on mathematical functions $\stackrel{\text { ? }}{?}$

\section{References}

Abrahams, M. (1967). Mechanical behaviour of tendon in vitro, a preliminary report. Medical and Biological $\overrightarrow{\mathbb{D}}$ Engineering, 5, 433-443.

Cronkite, A. E. (1936). Tensile strength of human tendons. Anatomical Record, 64, 173-186. 
Cruise, A. J. (1958). The structural periodicity of microscopic collagen fibres. Recent Advances in Gelatin and Glue Research, p. 45 Ed. by G. Stainsby. Pergamon, London.

Dale, W. C., Baer, E., Keller, A., and Kohn, R. R. (1972). On the ultrastructure of mammalian tendon. Experientia, 28, 1293-1295.

Diamant, J., Keller, A., Baer, E., Litt, M., and Arridge, R. G. C. (1972). Collagen ultrastructure and its relation to mechanical properties as a function of ageing. Proceedings of the Royal Society. Series $B, 180,293-315$.

Elliot, D. H. (1965). Structure and function of mammalian tendon. Biological Reviews of the Cambridge Philosophical Society, 40, 392-421.

Euler, L. (1744). De curvis elasticis. Methodus Inveniendi Lineas Curvas Maximi Minimive Proprietate Gaudentes. Lausanne.

Evans, J. H., and Barbenel, J. C. (1975). Structural and mechanical properties of tendon related to function. Equine Veterinary Journal, 7, 1-8.

Frisch-Fay, R. (1962). Flexible Bars. Butterworth, London.

Gathercole, L. G. J., and Keller, A. (1975). Light microscopic waveforms in collageneous tissues. Structure of Fibrous Bio-polymers. Colston Papers, Vol. 26, p. 153. Ed. by E. D. T. Atkins and A. Keller. Butterworth, London.

Gathercole, L. G. J., Keller, A., and Shah, J. S. (1974). Periodic wave pattern in native tendon collagen: correlation of polarising with scanning electron microscopy. Journal of Microscopy, 102, 95-105.
Harris, E. H., Bass, B. R., and Walker, L. B. (1964). Tensile strength and stress-strain relationships in cadaveric human tendon. Anatomical Record, 148, 289.

Harris, E. H., Walker, L. B., and Bass, B. R. (1966). Stressstrain studies in cadaveric human tendon and an anomaly in the Young's modulus thereof. Medical and Biological Engineering, 4, 253-257.

Haut, R. C., and Little, R. W. (1972). A constitutive equation for collagen fibres. Journal of Biomechanics, 5, 423-430.

Kear, M. (1971). The determination of mechanical strain in collageneous tissue during locomotion. Ph.D. dissertation, University of Bristol.

Lerch, H. (1950). Uber den Aufbau des Sehnengewebes. Gegenbaurs Morphologisches Jahrbuch, 90, 192.

Love, A. E. H. (1944). Theory of Elasticity, 4th ed. Dover, New York.

Rigby, B., Hirai, N., Spikes, J., and Eyring, H. (1959). The mechanical properties of rat-tail tendon. Journal of General Physiology, 43, 265-283.

Shaw, P. C. (1968). A method of flexor tendon suture. Journal of Bone and Joint Surgery, 50B, 578-587.

Tkaczuk, H. (1968). Tensile properties of human lumbar longitudinal ligaments. Acta Orthopaedica Scandinavica, Suppl. 115.

Verzár, F. (1957). The ageing of connective tissue. Gerontologia, 1, 363-369.

Viidik, A. (1973). Functional properties of collageneous tissues. International Review of Connective Tissue Research, Vol. 6, p. 127. Academic Press, New York. 